\title{
Three Thoughts in Art Research in Big Data Age
}

\author{
Weiwei Wang ${ }^{1}$ \\ ${ }^{1}$ Science and Technology College Gannan Normal University,Ganzhou,Jiangxi,341000,China
}

\begin{abstract}
With the arrival of big data era, its research methods, ideas, technology models and so on are constantly changing our learning, life and work model. Big data has made all walks of life enter a new stage of transformation. Most scholars at home and abroad believe that the technical essence of big data can be excavated through phenomena, which can be used as an effective means to eliminate disadvantages. However, it is widely known that, as a specialized subject to study human creative activities, laws and aesthetic manifestations, art research is influenced by the big data era, and a series of changes are taking place in the links of data extraction, research process and analysis of research results. Through the research, this paper combs out the thinking turn, method turn and art history view turn of art research under the background of big data, and puts forward the improvement strategy of art research, thus providing the direction for the development of art.
\end{abstract}

\section{Introduction}

The development of science and technology brings opportunities to various industries, but also makes them face more challenges. Art research is based on the collection of characters and images, through classification, screening, discrimination, textual research and a series of processing, so that the art research work can be carried out smoothly, is the main work form of art research at the present stage. However, under the influence of big data, Internet, artificial intelligence and so on, on the one hand, the progress of science and technology makes art research save a lot of manpower and material resources, save time and reduce cost, on the other hand, it also makes art research face great challenge and pressure[1].

\section{Three Choices in Art Research in Big Data Age}

Data is widely used in art research. In the field of art research, data can be divided into image data and text data according to different properties. These two kinds of data play a very important role in art research. However, due to the lack of scientific level and the lack of Internet for a long time, art researchers are much more difficult to obtain image data than to obtain text data. With the development of information technology, the functions of search, storage, analysis and integration in Internet platform make image data play a more and more important role in art research. In recent years, art galleries, museums and other public institutions with a large number of art image resources have begun to open the collection of high-definition image resources for the community[2]. For example, in the 2013 content opening program of the Getty Museum in the
United States, the museum will open 4600 high-definition pictures of the collection to users around the world free of charge. At the same time, the project does not limit the scope of download and use of users, but simply requires users to provide download reasons when downloading these images. The Getty Museum can not only get more data, but also analyze the user's behavior preferences and aesthetic principles. At the same time, these collected data will also provide more reference for the museum's educational activities, research and exhibition. Therefore, the project ultimately leads to a win-win outcome. In addition, the use of big data will make art research generally present the following three aspects of thinking turn[3].

Table1. The Thinking Turn of Art Research

\begin{tabular}{|c|c|c|}
\hline \multicolumn{4}{|c|}{ The Thinking Turn of Art Research } \\
\hline 1 & $\begin{array}{c}\text { The Turn of } \\
\text { Thinking }\end{array}$ & $\begin{array}{c}\text { The Transformation of Proof } \\
\text { Thinking to Digital Thinking }\end{array}$ \\
\hline 2 & Method change & $\begin{array}{c}\text { The Transformation of Causality } \\
\text { Judgment to Correlation Analysis }\end{array}$ \\
\hline 3 & $\begin{array}{c}\text { Changing View } \\
\text { of Art History }\end{array}$ & $\begin{array}{c}\text { Building History and Looking } \\
\text { Forward }\end{array}$ \\
\hline
\end{tabular}

\subsection{The Turn of Thinking: The Transformation of Evidence-based Thinking to Digital Thinking}

The history of art research is based on "things ", that is, taking objective things as the core, based on the substantive evidence of objective things, using the thinking mode of" hypothesis-verification "to study, which is a typical philosophical thinking. In this process of thinking, researchers rely too much on objective things and need to spend a lot of manpower and material resources to collect and update data, and the process of data analysis and collation has more subjective color. The

*Corresponding author's e-mail: 739960103@qq.com 
research results lack objectivity. In the middle of the 20th century, with the development of metrological research methods, a new mode of thinking was injected into the field of humanities and social sciences. At the present stage, influenced by the development of big data, advanced science and technology bring more possibilities for the scope, content and way of art research. Big data is not a superficial understanding of "big data" or "more data ", but a variety of data types, such as structured, semistructured data tables[4], various pictures, video, audio, geographical location and other data, which need to be stored, transmitted, analyzed, etc., technology, so as to break the space and time constraints of data resources, further improve the effectiveness of data utilization, and finally realize the transformation of data to digitization.
Under the background of big data, big data thinking regards data as a whole, and brings us unprecedented information value through the use of technical means. For example, Dunhuang grottoes, known as "the most valuable cultural discovery of the 20th century ", are designed by" digital Dunhuang "not only to collect, process and process information on grottoes, murals and other related cultural relics, but also to use big data means to analyze and organize massive literature, images, videos, data, etc., so as to construct an intelligent digital resource bank. In this resource bank, researchers can browse the panorama of grottoes in the way of human-computer interaction (as shown in figure 1), and can also search through images and Dunhuang vocabulary, and finally obtain key research data[5].

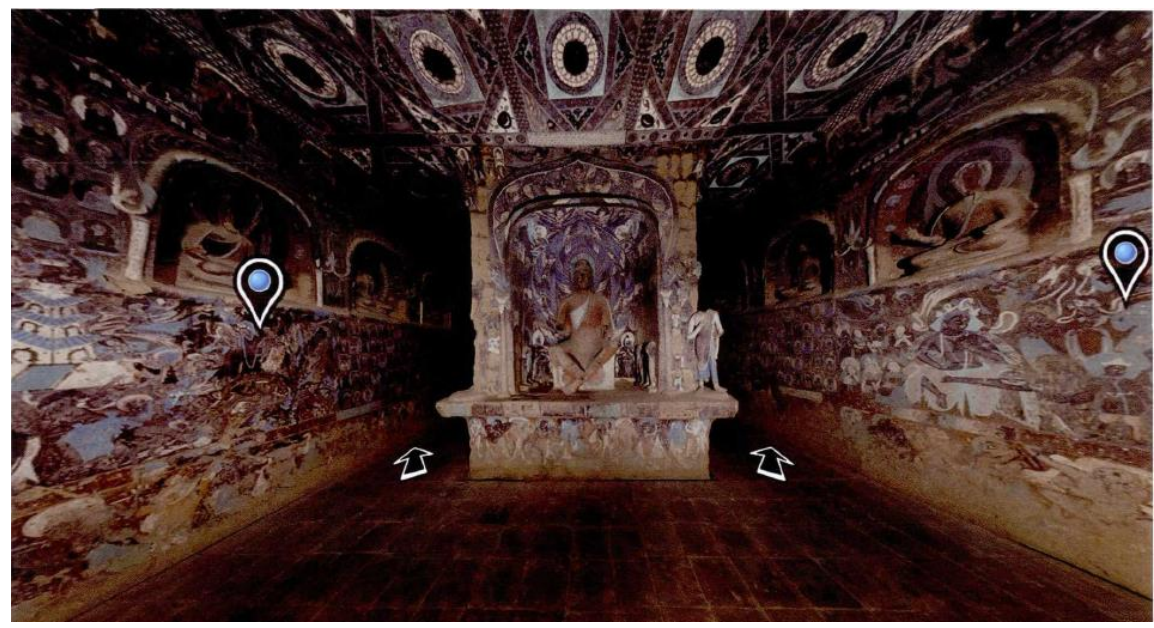

Fig 1. Panorama of the Grottoes

In addition, the digital construction of the Beijing Palace Museum enables most of the collection and cultural relics in the hospital to be retrieved and displayed on the digital platform (as shown in figure 2).

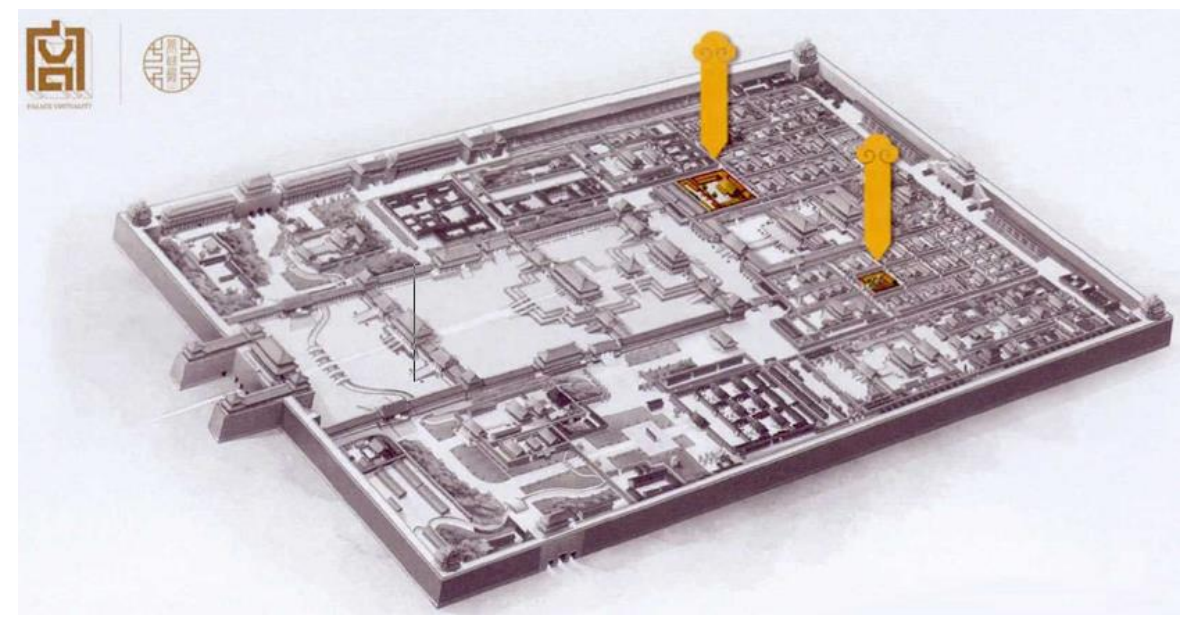

Fig 2. Beijing Palace Museum digital platform

\subsection{Method change : from Causal Judgment to Correlation Analysis}

The traditional art research work emphasizes the causality between the research elements and neglects its relevance. To know that before the conclusion of objective things, we should not only rely on logical evidence, but also carry out multi-party verification. The arrival of big data not only provides people with massive data resources, but also enables us to acquire the ability of association analysis in a complex cognitive world. In other words, art researchers began to pay attention to the value of data itself. Relying on big data platform and technology, art researchers 
gradually set up macro thinking and jump out of the "one leaf obstacle" thinking pattern, thus turning to the diversity, complexity and relevance of things.

Traditional art work usually only studies some specific artists or calligraphy and painting works of a specific period. In the background of big data, researchers can bring all artists and their works into the scope of research. Through data analysis and collation, we can effectively count the artists' identity background, family relationship, education, experience, interests, work information, work evaluation, quantity and other effective information. In other words, the art research under the background of big data is no longer based on the objective existence of art works, art style, theme, etc., but on the basis of the culture carried and inherited by the research works, a specific historical space-time network system can fully break the boundaries of various disciplines, time and space, endow art works with more historical, cultural, political and other values, and finally help researchers to see the essence of things through phenomena through the means of data.

\subsection{Changing View of Art History: Constructing History and Looking Forward}

Big data is quietly changing our cognitive style. On the basis of the traditional cognition of "material" and "culture ", we hope to seek the historical truth in the data through the collation and discrimination of historical data, so as to realize the objective remodeling of history. Under the background of big data, with the improvement of the degree of digitization of culture, art and history, people's historical concept has changed obviously, because big data makes history get rid of the boundary of time and space and literature[6].

At the same time, the relationship between people and big data is undergoing subtle changes, which will change people's understanding of reality. In the past, people recognized the world from the point of view of material and culture, and pursued the understanding of the superficial meaning of things. However, with the development of big data and the progress of science and technology, people are in an era of information explosion, and the world has become an all-encompassing and manageable category of information. In theory, big data contains a variety of massive data in human history, current and future social development. However, under the influence of information technology, people's thinking mode based on big data has gradually changed from historical concept to the possibility of creation. Thus, under the background of big data, people's view of history has been re-examined. At the same time, people's cognitive history of space and field also began to rebuild. This will provide a new recording method for the progress of human society and the development of human civilization[7].

\section{Cold Thoughts on Fine Arts Research in the Big Data Age}

Although big data brings us many opportunities and development, it also makes us experience a "worst time ". The problems of information flooding, failure, disorder, leakage and so on make the data extraction in art research difficult to distinguish between true and false, such as text and image database need to be further improved. Such as text database, take "Qi Baishi" as an example, take these three words as key words to search on the Internet, finally Baidu encyclopedia will show Qi Baishi related content, mainly including painting style, theory, life experience, works, disciples, social evaluation and so on, but the exhibition and collection donation of his works are not mentioned[8]. Although the introduction of online alignment of Baishi is still rich, in terms of aesthetic research, it is far from enough to rely on basic data, and there still a lot to be added.Not all countries have done poorly on their word base, and there is a site called "art facts" in the uk. The site's search page set up "artist "," exhibition "," agency" three search items, although not many items, but the content covered is very comprehensive. The biggest feature of the website is the collection and collation of artists is very comprehensive, the content not only includes the basic introduction, works, evaluation and so on, but also the organization of individual exhibitions, group exhibitions, joint exhibitions and other information are very detailed, which provides real and comprehensive information for art research.It can be seen that real and effective data information can not only help researchers reshape history, but also enable researchers to master historical laws, so as to better look forward to the future and establish a forward-looking view of art history, but incomplete or wrong information can only hinder or even mislead art research[9].

In addition, China's domestic database is not perfect. For example, China Zhiwang, it only has the traditional keyword search function. Even if the related papers included in the network already have a certain time span, but the paper document electronic still needs a certain time period. At the same time, China knows that the online collection of professional literature is not so comprehensive. Based on the perspective of researchers, the screening ability of the basic literature value of China knowledge Network is relatively lacking. In other words, compared with the open image data of museums mentioned above, China Zhiwang and other databases in China currently only have the most basic and lowest databases, which have not risen to the form of research. Therefore, for domestic researchers in China, they lack a professional database[10].

The establishment of professional form database can not be separated from the enthusiasm and participation of researchers. Traditional database development is supported by Internet search technology and lacks the needs of professional researchers. Therefore, the current art research, especially the art teaching in colleges and universities, should proceed more from the resources of the professional research institutions or the secondary combined with the specific needs of researchers, and 
constantly develop a professional database with its own characteristics. At the same time, research institutions and colleges and universities should establish a dynamic database update and sharing mechanism. In addition, art research can also promote the formation of professional databases through various exhibitions. For example, in recent years, the literature of artists has been widely loved by all walks of life. Each literature exhibition reviews the artist's individual literature and image data as a whole. Art researchers and institutions should pay attention to the accumulation of normal information, thus gradually forming a professional database[11].

\section{CONCLUSION}

The big data era provides opportunities for aesthetic research, but faces challenges at the same time. Under the background of big data, the continuous improvement of the basic database and the increasingly formed professional database will inevitably bring us a new perspective of art research. Art researchers should adapt to the development of the times and change their thinking in time. At the same time, art researchers should give full play to the platform and technical advantages of big data, avoid the disadvantages of information confusion, disorder, distortion and so on, and identify them through scientific collation in the massive information base, so as to create more possibilities for their own development.

\section{Author:}

Weiwei Wang(22 November 1981), gender: male, native: Longnan, Jiangxi, ethnic: Han, master's degree, lecturer, research field: fine arts, folk art.

\section{References}

1. Ma,X.D. (2017)A Cold Reflection on Fine Arts Research in the Big Data Era [J]. Art observation.

2. Meng, F.W.Zhu ,S.Li M.Y.(2017) A Study of Foreign Fine Arts in China in the 21st Century[J]. A Art observation.

3. Zhu,Y. (2019)Transformation and Innovation of Editing and Publishing in the Age of Data [J]. The study of transmission.

4. Zheng, Y. Shao,Y. Huang,X.F. Meng F.W.(2017)Discussion on Chinese Fine Arts Research from Edge to Self-conscious — in Foreign Countries $[\mathrm{J}]$. Art Observation

5. Li,J.(2016) A Study on the Innovative Development Path of Chinese Fine Arts Teachers' Education Based on Big Data Thinking [J]. Journal of Aesthetic Education.

6. He,J.Y.(2018)The Intervention of Big Data in Fine Arts Research.The Intervention of Big Data in Fine Arts Research.

7. Wu,H.B.(2017)Three Thoughts in Art Research in Big Data Age.Art Observation.
8. Liu,J.S.(2016)Paradigm Reflection and Methodology Construction of Regional Art Research.Regional Studies on Culture and Literature.

9. Chen,W.A.(2014)An Island of Art Research in the Global Age — Taking Lingnan Regional Art Research as an Example.Chinese Painting Journal.

10. Wang,R.(2014)The Dilemma and Self-construction of Foreign Art Research in China.Art Observation.

11. Zhu,L.(2014)Some Thoughts on the Present Situation of Art Research and Publication.Art Observation. 